\title{
Organophosphate poisoning in pregnancy: Is it a cry for help?
}

\author{
S N Cebekhulu, ${ }^{1}$ MB ChB, Dip Obst (SA), MMed (O\&G), FCOG (SA); R C Pattinson, ${ }^{2}$ MD, FRCOG, FCOG (SA)
}

${ }^{1}$ Department of Obstetrics and Gynaecology, Kalafong Provincial Tertiary Hospital, and Faculty of Health Sciences, University of Pretoria, South Africa ${ }^{2}$ Medical Research Council Maternal and Infant Health Care Strategies Unit, Department of Obstetrics and Gynaecology, Faculty of Health Sciences, University of Pretoria, South Africa

Corresponding author: S N Cebekhulu (sylvia.cebekhulu@up.ac.za)

Organophosphate poisoning seems to be a fairly common cause of mortality in young and teenage mothers. The attending healthcare personnel, especially nursing staff and doctors, should always have a high index of suspicion when attending to teenagers who have never initiated antenatal care or those who request termination of pregnancy (TOP), because often - if these patients are not assisted - they choose unsafe, unauthorised alternatives, which could have dire consequences. A management plan should always include preventive measures, attempts to diagnose, and prompt, directed immediate and maintenance therapies. Preventive measures must include clear decisive plans on how to manage women who request TOP and advocacy for use of long-acting reversible contraception, including the intra-uterine device, for all young women who are at any time in contact with the health system. Early and correct diagnosis is mainly guided by the history, including a collateral history if the woman is unresponsive. Attending health personnel are usually able to establish substance ingestion accompanied by a varying degree of symptoms - from nausea, vomiting, hypersecretions, headache, severe confusion, pinpoint pupils, bradycardia, tachypnoea/bradypnoea, hypertension/hypotension to convulsions, coma and eventually death. The basic concepts of multidisciplinary team resuscitation, including the Airway, Breathing, Circulation, Disability, Exposure (ABCDE) approach, should always be followed to stabilise the patient, while trying to elicit the cause and thus assist with further management.

S Afr Med J 2018;108(11):907-909. DOI:10.7196/SAMJ.2018.v108i11.13407

Acute and intentional poisoning is one of the most common reasons why pregnant and non-pregnant patients present to the emergency department of a health service. Young patients are most commonly affected, with a high mortality rate. In most instances, the ingested agent(s) is unknown. Only the victim knows what happened, and at presentation they might be unable to communicate the information. The first responder is the second-best person to identify the poison, which rarely occurs. Agitation that progresses to central nervous system depression and subsequent coma is common in the majority of toxin cases.

The files of women classified as coincidental deaths by the National Committee on Confidential Enquiries into Maternal Deaths were examined to establish if those who might have died from poisoning could be identified. Some examples are presented. Organophosphate ingestion appeared to be a fairly common cause of poisoning that resulted in maternal deaths. Signs and symptoms of such poisoning are discussed.

\section{Case 1}

A teenage primigravida presented to her local clinic during the first trimester of pregnancy, requesting termination of pregnancy (TOP). No management plan was prepared regarding her request, despite repeated visits. She was also HIV-positive, her CD4 count was unknown and she started antiretroviral treatment. Three months later the acutely ill patient presented at a tertiary hospital and was admitted to the antenatal ward. She was administered fluids and antibiotics, but she progressively deteriorated. She was then transferred to a high-dependency ward and died within an hour of admission from multi-organ (renal, respiratory, haematological and metabolic) failure secondary to suspected poisoning.

\section{Lessons learnt}

- Take requests for TOP seriously, construct clear management plans and document them. Involve a social worker.

- Do not underestimate the clinical condition of a patient in respiratory distress.

\section{Case 2}

A teenage primigravida who had not attended antenatal care was admitted to a district hospital 6 hours after taking an overdose of a known substance. She was clinically in shock and was admitted for 3 days; her condition became progressively worse. She died on the third day while waiting for a transfer to a higher level of care. During the time at the district hospital, she mainly received nursing care and occasional telephonic advice from a doctor.

\section{Lessons learnt}

- Do not delay referral of critically ill patients.

- Request doctors to personally review ill patients.

\section{Case 3}

A young woman in her early twenties, who previously delivered a baby by caesarean section, was now in her third trimester and received no antenatal care. She was found dead at home after carbamate ingestion.

\section{Lesson learnt}

- Emphasise the importance of (long-acting) contraception post partum and during every contact with the health system.

\section{Case 4}

A 20 -year-old pregnant woman who had not attended antenatal care 
presented at a hospital with a history of vomiting, diarrhoea and collapse. On arrival, she had pinpoint pupils and she was unresponsive, with copious secretions and bilateral chest crepitations. The patient was initially normotensive, but suddenly became hypotensive and bradycardic. She was intubated and transferred to a higher level of care, where she died shortly after arrival from suspected organophosphate poisoning.

\section{Lessons learnt}

- Stabilise the patient before referral.

- Know the initial steps of stabilisation for organophosphate poisoning.

\section{Discussion}

- Organophosphates were first synthesised during the 19th century by French chemist Louis Lassaigne. During World War II, the German chemist, Gerhard Schrader, synthesised many commercial insecticides and chemical warfare agents, such as tabun and sarin. Shortly after the war, organophosphates were used widely as pesticides. ${ }^{[1]}$

- Organophosphates are easily accessible and self-poisoning is an important clinical problem in rural regions of the developing world, with $>200000$ deaths yearly.

- They can be absorbed through the skin, lungs and gastrointestinal tract. $^{[2,3]}$

\section{Pathogenesis and clinical presentation}

- Organophosphates act mainly by inhibiting acetylcholinesterase action, thereby leading to accumulation of acetylcholine and subsequent overstimulation of muscarinic and nicotinic receptors.

- The degree of neurotoxicity and severity can be determined by direct measurement of the true (red blood cell) cholinesterase activity and can be classified as mild ( $>40 \%$ activity), moderate (20 - 40\% activity) and severe ( $<20 \%$ activity).

- Patients may present with varying degrees of clinical symptomatology, ranging from nausea, vomiting, hypersecretions (bronchorrhoea, urination, diarrhoea), headache, severe confusion, pinpoint pupils, bradycardia, tachypnoea/bradypnoea, and hypertension/hypotension to convulsions, coma and eventually death. ${ }^{[4]}$

\section{Natural progression after exposure}

Clinical phases of organophosphate poisoning include the following:

- Acute cholinergic crisis - within 24 hours after the initial presentation, and described in patient 4.

- Intermediate syndrome - typically occurs within 24 - 96 hours and is dependent on the reversibility of enzyme blockade. This can also affect the consciousness of patients without cholinergic signs or those who were apparently successfully treated for acute cholinergic crisis. It involves paralysis of the muscles of respiration, proximal limb muscles, neck flexors and muscles innervated by motor cranial nerves. Appropriate therapy that commonly includes artificial respiration yields complete recovery 5 - 18 days later. ${ }^{[5]}$

- Delayed neuropathy - involves both the peripheral and central nervous systems. It is characterised by motor and sensory

Table 1. Acute/immediate management of organophosphate poisoning

\begin{tabular}{ll}
\hline Management & Intervention \\
\hline Airway & Stabilisation \\
& Intubate if threatened airway or unconscious \\
& Be ready for difficult airway
\end{tabular}

Comments

Protects against aspiration

Drug interactions may occur with certain toxins; therefore, use of some agents, e.g. suxamethonium, is contraindicated because of delayed action from unavailable cholinesterase enzyme for its metabolism

Breathing

High-flow oxygen, invasive or non-invasive ventilatory support

Circulation

Rapid infusion with isotonic solutions, including vasopressors/inotropes, should be timeously considered

Appropriate laboratory testing, including cholinesterase levels, glucose levels, blood count, urea and electrolyte levels, acetaminophen and salicylate levels, and other specific drug and enzymes levels Comprehensive urine drug testing

Disability and neurological stabilisation Assessment by AVPU (Alert, response to Voice, response to Pain and Unconscious) should be performed immediately and patients appropriately managed

Seizures are best aborted with benzodiazepines Administer propofol if benzodiapines are ineffective

Exposure and elimination

Remove soiled clothing and containers and wash with soap containing $30 \%$ ethanol

Other
Identify any injuries

Gastric lavage

Activated charcoal

Haemodialysis
Respiratory parameters, e.g minute ventilation; oxygenation should guide the use of these interventions Define targets, e.g. urine output $(\geq 0.5 \mathrm{~mL} / \mathrm{kg} / \mathrm{h})$, lactate clearance, mean arterial pressure

Guides multiple-agent ingestion, degree of organ involvement and directed treatment modalities

Rapid deterioration is highly possible

There might be other causes of seizures, and specific antidotes may be necessary, e.g. pyridoxime for isoniazid poisoning

Reduces risks to healthcare workers and continued patient exposure

Only after stabilisation and treatment with oxygen, atropine and oximes may be administered No mortality benefit due to rapid absorption of pesticide into blood

No clear role 
neuropathies. Rehabilitation programmes are usually necessary for full recovery.

\section{Management}

In severe poisoning, organised targeted resuscitation is mandatory and can be lifesaving:

- The basic concepts of multidisciplinary team resuscitation (Airway, Breathing, Circulation, Disability, Exposure (ABCDE)) should always be followed (Table 1). The goals are to stabilise the patient, while trying to elicit the cause, and thus assist with further management.

- There are common syndromes associated with different poisonous agent(s), such as sympathomimetics, opioids, sedatives, cholinergics, anticholinergics, hallucinogens and serotonins.

- Patients might gain access to any of these abovementioned agents. Initially it may not be possible to exclude poisoning with multiple agents.

- Immediate intravenous access and cardiac and respiratory monitoring should be implemented.

- Clinical signs and symptoms guide management of these patients in most instances, as testing for poisons is not always readily available.

- Treatment includes atropinisation (initiated as a rapid bolus of 2.0 - $4.0 \mathrm{mg}$ atropine followed by $0.5-2.0 \mathrm{mg}$ every 10 - 15 minutes, or by infusion until atropinisation is achieved, and a subsequent maintenance dose given thereafter; this can be administered for several days to achieve full recovery).

- It is important to define targets, such as improved secretions, pupil size and pulse response, to guide atropinisation and detect atropine toxicity.

- In situations where there is doubt about the need for atropine, a test can be performed, whereby $1.0 \mathrm{mg}$ atropine is given. The response (tachycardia with skin flushing) will indicate whether further administration of the agent is indicated.

- The use of pralidoxime to reactivate cholinesterase is controversial and influenced by many factors, such as rate of inhibition and cholinesterase ageing. However, the World Health Organization recommends its use in patients requiring atropine and those with evidence of cholinergic toxicity, neuromuscular dysfunction and exposure to agents with delayed neurotoxicity.

- Symptomatic systemic support (Table 1) is mandatory.

Very importantly, prolonged resuscitation efforts are usually not futile in these patients and previously healthy patients have a better prognosis. $^{[6-9]}$

\section{Subsequent management}

- Multidisciplinary (should include a toxicologist, poison centre personnel, physician, intensivist, obstetrician and gynaecologist, psychologist, social worker) teamwork is involved regarding definitive diagnosis, progressive, co-ordinated weaning of acute treatment, subsequent de-escalation of care and post-intensive care unit management.

- There should be monitoring and management of complications.

- Attention should be given to the patient's psychosocial stressors to prevent repeated poisoing attempts.

\section{Organophosphates in pregnancy}

- Not well studied or reported.

- Mainly retrospective cases - the majority of patients received atropine and ventilator care.

- There were no immediate congenital abnormalities or neurological deficits in babies.
- The majority of maternal deaths occurred during the acute cholinergic crisis and intermediate syndrome phases of organophosphate poisoning. ${ }^{[10]}$

\section{Summary}

- Unplanned and unwanted pregnancies in young women are a reality.

- Young women with organophosphate poisoning have usually not attended antenatal care and/or have a history of a previous request for TOP.

- Organophosphates are easily accessible and a high index of suspicion should be maintained in these young women.

- The initial diagnosis may not be obvious; however, clinical signs of mental alteration (confusion, headaches, restlessness and possibly collapse), pinpoint pupils and hypersecretions (diarrhoea, urination, bronchorrhoea) with shortness of breath or respiratory distress should alert the clinician regarding the diagnosis of organophosphate poisoning.

- The initial assessment, $\mathrm{ABCDE}$ and management with appropriate system support, including rapid atropinisation and early mechanical ventilation, are mandatory.

- Ingestion of more than one toxin may be a possibility.

- Be prepared for delayed presentation after recovery from the acute cholinergic crisis.

We should look for the 'she's': 'She says she's fine, but she's going insane. She says she feels good, but she is in a lot of pain. She says it is nothing, but it is really a lot. She says she is okay, but really she's not.' Many lives could be saved if detected early by primary healthcare personnel, mainly doctors!

Declaration. None.

Acknowledgements. The authors wish to express their gratitude to the chairperson of the National Committee for Confidential Enquiries into Maternal Deaths in SA, Prof. J Moodley, for allowing the assessment of the cases, and to the SA Medical Research Council and the University of Pretoria for support.

Author contributions. As part of the secondary analysis permitted by the National Committee for Confidential Enquiries into Maternal Deaths, there was a striking and disturbing observation of how mothers, whose cause of death was classified as coincidental, died so soon. This deserved an introductory analysis; hence, a few cases of poisoning were reviewed with the intention of creating a platform for awareness and future in-depth analysis of these deaths. Both authors equally contributed to this article.

Funding. None.

Conflicts of interest. None.

1. Van Horing DJ, Veale DJH, Muller GF, et al. Emergency management of acute poisoning. Afr J Emerg Med 2001;1:69-78. https://doi.org/10.1016/j.afjem.2011.07.006

2. Petroianu GA. History of organophosphates synthesis: The very early days. Pharmazie 2010;65:306311. https://doi.org/10.1691/ph.2010.9141

3. World Health Organization/United Nations Environment Programme. Public Health Impact of World Health Organization/United Nations Envirom
Pesticides used in Agriculture. Geneva: WHO, 2007.

4. Namba T, Nolte CT, Jackrel JJ, et al. Poisoning due to organophosphate insecticides: Acute and chronic manifestations. Am J Med 1971;50(4):475-492. https://doi.org/10.1016/0002-9343(71)90337-8

5. Noshad H, Ansarin K, Ardalan MR, et al. Respiratory failure in organophosphate insecticide poisoning. Saudi Med 2007;28(3):405-407.

6. Ram JS, Kumar SS, Jayarajan A, et al. Continuous infusion of high doses of atropine in the management of organophosphorus compound poisoning. J Assoc Phys India 1991;39(2):190-193.

7. Wood DM, Wyncoll DLA. Management of acute poisoning. Oh's Intens Care Manual 2014;88:892-902.

8. Eddleston M, Karalliedde L, Dawson AH, et al. Early management after self-poisoning with an organophosphorus or carbamate pesticide - a treatment protocol for junior doctors. Crit Care 2004;8:R391-R397. https://doi.org/10.1186/cc2953

9. Eddelston M, Buckley NA, Eyer P, et al. Management of acute organophosphorus pesticide poisoning Lancet 2008;371:597-607. https://doi.org/10.1016/50140-6736(07)61202-1

10. Adhikari $\mathrm{K}$, Ghosh A, Alauddin MD, et al. Organophosphate poisoning in pregnancy. J Obstet Gynaecol 2011;31(4):290-292. https://doi.org/10.3109/01443615.2010.545901 